\title{
Vendor and retailer managed consignment inventory with additive price-dependent demand
}

\section{Milena Bieniek ${ }^{1}$}

Received: 11 January 2018 / Accepted: 7 November 2018 / Published online: 20 November 2018

(c) The Author(s) 2018

\begin{abstract}
Consignment is the shifting of the inventory ownership to the supplier. In this form of business arrangement the supplier places goods at a customer's location without receiving payment, until the goods are sold. We consider a single period supply chain model, where the supplier contracts with the retailer. Market demand for the product is price-dependent and uncertain. The supplier decides the consignment price and the retailer chooses the retail price for each unit sold. Two arrangements called retailer managed consignment inventory (RMCI), and vendor managed consignment inventory (VMCI) are studied. The only difference between these arrangements is that under RMCI contract the retailer is allowed to choose the service level, and under VMCI contract the supplier decides about this service level. In our paper we give the optimal solutions for the retail price, the service level and the consignment price in closedform, which maximize the expected profit of the retailer or the supplier under both consignment regimes. We consider the additive demand linearly dependent on price. We also illustrate the solutions by a numerical example, which explains the general results well.
\end{abstract}

Keywords Consignment · Inventory control · Additive demand

\section{Introduction}

Supply chain management has been one of the major tasks for management professionals. The top practice for reducing the inventory cost is using the consignment, which is shifting the inventory ownership to the suppliers, until the goods are used or sold. More precisely, it is the process of placing goods in the retailer's location and no payment is made to the supplier before the item is sold. Hence, the retailer faces lower

Milena Bieniek

milena.bieniek@umcs.lublin.pl

1 Economic Faculty, Maria Curie Sklodowska University, Plac Marii Curie-Sklodowskiej 5, 20-031 Lublin, Poland 
risk associated with uncertain demand, since he has no money tied up in inventory. How much inventory to hold, can be chosen by the retailer or the supplier. These arrangements are called retailer managed consignment inventory (RMCI) and vendor managed consignment inventory (VMCI), respectively. Both consignment contracts have their applications in practice but in recent years VMCI has became to be more and more popular. Using VMCI contract is especially popular in on-line stores such as Amazon.com or eBay.com. Besides, VMCI is implemented in many big retailers like Wal-Mart, Meijer Stores and by the computer manufacturers. However, many practitioners prefer to apply mixed arrangements and still debate, which arrangement is better for the channel participants (cf. [8]).

In our paper we focus on VMCI and RMCI settings introduced in Ru and Wang [10]. In the mentioned paper the authors build Stackelberg type game-theoretic model to capture the interactions between the supplier and the retailer when one of them controls the supply channel inventory. Market demand for the product is random and price-sensitive. Both the supplier and the retailer incur a linear cost for producing and handling the product. The supplier offers the consignment price charged to the retailer before the demand is realized. Moreover, at the same time, the retailer chooses the retail price for selling the product to the market. Two consignment settings are considered, which are dependent on the fact, who makes the decision about the supply chain inventory or equivalently about the customer service level. Under RMCI contract the retailer chooses the inventory level complete with the selling price. Under VMCI contract the supplier decides on the inventory level together with the consignment price.

In the paper of Ru and Wang [10] the authors adopted multiplicative function of the demand with exponential function of the price. Using this special form of the demand let them give the closed-form analytical solutions. The authors also point out that using linear price-dependent demand model might cause difficulties for giving precise mathematical results. Despite of this statement we solve that problem for the additive demand with linear function of the price. Namely, we present the exact solutions for described consignment models. In particular RMCI contract is challenging and mathematical proofs are much more complicated than for VMCI and finally for RMCI setting with multiplicative demand.

Our article is the extended version of the paper of Bieniek [2]. In detail the differences of Bieniek [2] and this study are as follows. We add the assumption on the distribution function of the random part of the demand to have the increasing failure rate property (IFR). It allows us to give the assumptions of the theorems in a simpler form, which can be checked more easily. In detail, in the former short version of our paper we use the limitation on lost sales rate elasticity to be less than or equal to a half on the whole domain. Now we transform it to the simple condition involving model parameters. We give also complete and precise solution for RMCI arrangement. We introduce some constraints under which this exact solution could be given. Moreover, we analyze the numerical example. In given example we use that kind of distribution, which satisfy the assumptions of the established theorems and lemmas. We present in the tables the results on the optimal quantities and we can observe the variability of the optimal service level, retailer price, consignment price, the retailer's and supplier's expected profits with respect to the share of channel cost. Finally, we show 
some figures of the channel profits and also the profit loss of the channel profit for both settings. It is worth to emphasize, that the existence of precise solutions put some limitations on the share of the channel cost.

In the following we provide a review of the papers that are closely related. The research presented here is relevant to the consignment contract with inventory ownership. Lee and Chu [8] address the issue, who should control the supply chain. In their consignment settings the wholesale and the consignment prices are exogenously given. In Wang et al. [11] the authors consider the single-product consignment contract with revenue sharing between the supplier and the retailer. This is a pure VMCI consignment arrangement, in which the supplier retains ownership of the inventory and bears all risk of overstocking. The retailer specifies the percentage allocation of sales revenue and, at the same time, the supplier chooses the product quantity and the retail price. In their model an iso-elastic demand curve is used. The recent papers treating also revenue-sharing consignment contracts are among others Feng et al. [4], Hu et al. [6] or Cai et al. [3]. The papers which extend the results of [10] and consequently are connected with our paper are $\mathrm{Hu}$ et al. [5] and [6]. In $\mathrm{Hu}$ et al. [5] VMCI and RMCI regimes with consumer returns and price-dependent exponential demand function are studied. In Hu et al [6] the authors compare VMCI arrangement with the revenue-sharing consignment for an iso-elastic multiplicative demand function.

\section{General assumptions and centralized channel decisions}

We consider a single-period supply chain in which the supplier (vendor) produces and sells a product to the retailer. The supplier decides his consignment price $w$, charged to the retailer for each unit sold. The retailer chooses retail price $p$ for selling the product to consumers. Denote by $c_{s}$ the supplier's unit production cost and by $c_{r}$ the retailer's unit handling cost. Also define $c=c_{s}+c_{r}$ as the total unit cost for channel and $\alpha=c_{r} / c$ as the share of channel cost that is incurred by the retailer. The random demand is defined by $D(p, \epsilon)=y(p)+\epsilon$, where $y(p)=a-b p, a, b>0$. Here $\epsilon$ is continuous random variable with the expected value $\mu$, the cumulative distribution function $F($.) and the probability distribution function $f($.) with the support $[A, B]$, where $A<0$ and $B>0$.

The general assumptions are

1. $c<p<p_{\text {max }}$, where $p_{\max }=\max _{p: y(p)+A>0}=\frac{A+a}{b}$

2. $A+a-b c>0$

The assumptions guarantee that realization of the demand $D(p, \epsilon)$ is positive.

For a centralized channel the decision maker has the ability to decide on the quantity to buy and the price to set for the good he sells. Such a decision is based on maximizing the expected channel profit given by:

$$
\Pi_{c}(p, Q)=p E(\min (D(p, \epsilon), Q))-c Q .
$$

We define $z=Q-y(p)$ and transform $\Pi_{c}(p, Q)$ to

$$
\Pi_{c}(p, z)=p \mu(z)+p y(p)-c(z+y(p)),
$$


where $\mu(z)=\mu+\int_{z}^{B}(z-u) f(u) d u$. As indicated in Petruzzi and Dada [9] the quantity $z$ can be interpreted as a safety stock because for selected value of $z$ we face shortages if $z<\epsilon$ or leftovers if $z>\epsilon$. On the other hand $z$ corresponds to a unique customer service level, which is given by

$$
P(D(p, \epsilon) \leq Q)=P(\epsilon \leq Q-y(p)=z)=F(z)
$$

Indicating the value for $z$ is equivalent to setting up the customer service level for the system.

Understanding the variability of the function $\mu(z)$ from (1) is crucial for the next analysis. The following statements hold:

1. $\frac{d \mu(z)}{d z}=1-F(z)$;

2. $\mu($.$) is an increasing function of z \in[A, B]$;

3. $\mu(A)=A<0$ and $\mu(B)=\mu$.

Then after some changes the objective function has the form:

$$
\max _{p, z} \Pi_{c}(p, z)=\max _{p, z}(p(\mu(z)+a-b p)-c(z+a-b p))
$$

To solve this problem we consider the sequential optimization method. This is the way of seeking optimum of a function of several variables be selecting the optimal values of each variable. Finally this method produces the maximum of the function we needed. We use this method and find the optimal solution denoted by $\left(p_{c}^{*}, z_{c}^{*}\right)$ to the problem of maximizing the central channel profit. The result needs some new definitions and assumptions.

Definition 1 (cf. [7]) The lost sales rate elasticity (LSR) for a given price $p(z)$ and service level $z$ for additive price-dependent demand is defined as

$$
\kappa(p(z), z)=\frac{b p(z) f(z)}{1-F(z)} .
$$

It was proved in Bieniek [2] that maximizing centralized channel profit (2) we get the following. For any given service level $z \in[A, B]$

1. the unique optimal selling price $p_{c}^{*}$ maximizing $\Pi_{c}$ is given by

$$
p_{c}^{*}(z)=\frac{\mu(z)+a+b c}{2 b},
$$

2. $p_{c}^{*}(z)$ is increasing and concave with $z, c<p_{c}^{*}(z)$, and also $p_{c}^{*}(z)<p_{\max }$ if

$$
2 A+a-b c>0 .
$$

If the assumption (2) is not satisfied then the solution $p_{c}^{*}$ can be suboptimal and the optimal expected profit can be underestimated. Further consideration needs to recall some characteristics on LSR elasticity. 
Lemma 1 (cf. [7]) For any given service level $z$, if $f($.$) satisfies the property of increas-$ ing failure rate (IFR) then LSR elasticity $\kappa\left(p_{c}^{*}(z), z\right)$ is increasing function of $z$, where $z \in[A, B]$.

Remark 1 Increasing failure rate, which means that $f(x) /(1-F(x))$ being increasing in $x$, is a very mild restriction on the demand distribution. This is satisfied by most commonly used probability distributions including normal, truncated normal, lognormal, uniform and exponential distributions (cf. [1]).

Using the above notions we get the theorem in which we specify the optimal service level. The proofs of all theorems and lemmas are put in the Appendix.

Theorem 1 If $f($.$) satisfies the property of increasing failure rate (IFR) and if$

$$
(A+a+b c) f(A) \geq 1
$$

then the optimal service level $z_{c}^{*}$ is unique root of the equation:

$$
\frac{\mu\left(z_{c}^{*}\right)+a+b c}{2 b}=\frac{c}{1-F\left(z_{c}^{*}\right)} .
$$

Remark 2 The assumptions of this theorem are different than those given in Bieniek [2]. Condition (3) can be more easily examined than the restriction on LSR elasticity $\kappa\left(p_{c}^{*}(z), z\right) \geq 1 / 2$ in the whole domain of the function under study, which was assumed in Bieniek [2]. However, (3) is still very restrictive since it assures concavity. It is sufficient but not necessary condition to guarantee that $z_{c}^{*}$ is an optimal solution. Obviously, if $\Pi_{c}(z)$ is not concave function of $z$, then $z_{c}^{*}$ could be the optimal solution.

\section{Decentralized channel decisions under $\mathrm{RMCl}$ contract}

\subsection{Retailer's retail price and service level decision}

Under RMCI contract decisions are made in two sequential steps. In step 1 the supplier specifies the consignment price to determine the amount of payment he will receive from the retailer for each unit of his product sold. In step 2 the retailer decides the quantity for the supplier to deliver and the retail price for selling the product to the market. By the sequential method we assign the selling price and the service level, which maximize the retailer's expected profit. The retailer's expected profit is given by

$$
\Pi_{d, R}(p, Q \mid w)=(p-w) E(\min \{D, Q\})-c \alpha Q
$$

or equivalently

$$
\Pi_{d, R}(p, z \mid w)=(p-w)(\mu(z)+a-b p)-c \alpha(z+a-b p) .
$$


It was proved in Bieniek [2] that for any given $z \in[A, B]$ and known $w>0$ the unique optimal retail price $p_{d}^{*}$, which maximizes the retailer's expected profit is given by

$$
p_{d}^{*}(z)=\frac{\mu(z)+a+b c \alpha+b w}{2 b} .
$$

It can be obtained from the equality $\frac{\partial \Pi_{d, R}(p, z \mid w)}{\partial p}=0$, since by $\frac{\partial^{2} \Pi_{d, R}(p, z \mid w)}{\partial p^{2}}<0$, $\Pi_{d, R}(p, z \mid w)$ is concave. Furthermore, by $\frac{d p_{d}(z)}{d z}=\frac{1-F(z)}{2 b}>0$ and $\frac{d^{2} p_{d}(z)}{d z^{2}}=$ $-\frac{f(z)}{2 b}<0$, the optimal price $p_{d}^{*}(z)$ is increasing and concave.

To prove next theorem we need the following lemma.

Lemma 2 For any given service level $z$ and a given $w$ such that $0<w<p_{d}^{*}(z)$, if $f($.) satisfies the increasing failure rate (IFR) property then LSR elasticity $\kappa\left(p_{d}^{*}(z)-w, z\right)$ is increasing function of $z$ for all $z \in[A, B]$.

Using the above statement we get the optimal service level.

Theorem 2 For consignment price $w>0$, if $f($.) satisfies the property of increasing failure rate (IFR) and, if

$$
A+a-b(c \alpha+w)>0
$$

and

$$
(A+a+b(c \alpha-w)) f(A) \geq 1
$$

then the optimal service level $z_{d}^{*}$ that maximizes $\Pi_{d, R}\left(p_{d}^{*}(z), z \mid w\right)$ is uniquely determined by

$$
\frac{\mu\left(z_{d}^{*}\right)+a+b c \alpha}{2 b}-\frac{w}{2}=\frac{c \alpha}{1-F\left(z_{d}^{*}\right)} .
$$

\subsection{Supplier's consignment price decision}

It should be emphasized, that in the case of additive demand both the optimal selling price $p_{d}^{*}(z)$ and the optimal service level $z_{d}^{*}$ are dependent on the consignment price $w$. It produces many difficulties for obtaining closed-form solutions. It is worth to note that for multiplicative demand, which is used in Ru and Wang [10] the optimal service level does not depend on the optimal consignment price and the derivations are simpler than here. The authors adopt a specific demand function form for the convenience of getting precise solutions. The problem considered with linearly pricedependent demand in RMCI contract needs much more attention. For instance we should give in the theorems some additional assumptions.

In step 1 of RMCI arrangement knowing that the retailer chooses $\left(p_{d}^{*}, z_{d}^{*}\right)$, the supplier's unique optimal consignment price $w^{*}$ can be calculated by maximizing the expected supplier's profit. This expected profit is given by: 


$$
\Pi_{d, S}\left(w \mid p_{d}^{*}, z_{d}^{*}\right)=w\left(\mu\left(z_{d}^{*}\right)+a-b p_{d}^{*}\right)-c(1-\alpha)\left(z_{d}^{*}+a-b p_{d}^{*}\right)
$$

Note that both $p_{d}^{*}$ and $z_{d}^{*}$ depend on $w$ and therefore we get

$$
\begin{aligned}
& \frac{\partial \Pi_{d, S}\left(w \mid p_{d}^{*}, z_{d}^{*}\right)}{\partial w}=w\left(\frac{d z_{d}^{*}}{d w} \frac{\left(1-F\left(z_{d}^{*}\right)\right)}{2}-b\right) \\
& -\frac{d z_{d}^{*}}{d w} \frac{(1-\alpha)\left(1+F\left(z_{d}^{*}\right)\right)}{2}+\frac{a+\mu\left(z_{d}^{*}\right)+b c(1-2 \alpha)}{2}
\end{aligned}
$$

where $z_{d}^{*}$ is defined by (6). Now our aim is to find the formula for $\frac{d z_{d}^{*}}{d w}$.

Lemma 3 If $f($.$) satisfies the property of increasing failure rate (IFR) and if$

$$
2 b c \alpha f(A)>1
$$

then the derivative $\frac{d z_{d}^{*}}{d w}$ exists and it is given by the formula

$$
\frac{d z_{d}^{*}}{d w}=\frac{b\left(1-F\left(z_{d}^{*}\right)\right)^{2}}{\left(1-F\left(z_{d}^{*}\right)\right)^{3}-2 b c \alpha f\left(z_{d}^{*}\right)}
$$

The lemma 3 is used in the next theorem in which the formula for the optimal consignment price is obtained.

Theorem 3 If $f($.$) satisfies the property of increasing failure rate (IFR) and the$ assumption (8) holds then the unique optimal consignment price $w^{*}$ is given by

$$
\begin{aligned}
w^{*}= & w^{*}\left(z_{d}^{*}\right)=\frac{1}{b\left(\left(1-F\left(z_{d}^{*}\right)\right)^{3}-4 b c \alpha f\left(z_{d}^{*}\right)\right)}\left(\left(\left(1-F\left(z_{d}^{*}\right)\right)^{3}-2 b c \alpha f\left(z_{d}^{*}\right)\right)\right. \\
& \left.\cdot\left(\mu\left(z_{d}^{*}\right)+a+b c(1-2 \alpha)\right)-b c\left(1-F\left(z_{d}^{*}\right)\right)^{2}\left(1+F\left(z_{d}^{*}\right)\right)(1-\alpha)\right) .
\end{aligned}
$$

We get

$$
p_{d}^{*}=\frac{2 b c \alpha f\left(z_{d}^{*}\right)\left(3 \mu\left(z_{d}^{*}\right)+3 a+b c\right)-\left(1-F\left(z_{d}^{*}\right)\right)^{3}\left(\mu(z)+a+b c(1-\alpha)-\frac{b c(1-\alpha)}{1-F\left(z_{d}^{*}\right)}\right)}{b\left(4 b c \alpha f\left(z_{d}^{*}\right)-\left(1-F\left(z_{d}^{*}\right)\right)^{3}\right)} .
$$

In case of RMCI contract the assumption $p_{d}^{*}<p_{\max }=\frac{A+a}{b}$ should be satisfied to omit the influence of the negative demand. But for the reason of complexity of the formulas one may check if it holds after he assigns the numerical value of $p_{d}^{*}$.

Finally, we can state that under RMCI contract the total decentralized optimal channel profit is equal to

$$
\Pi_{d}^{*}=\Pi_{d, R}^{*}+\Pi_{d, S}^{*}=p_{d}^{*}\left(\mu\left(z_{d}^{*}\right)+a-b p_{d}^{*}\right)-c\left(z_{d}^{*}+a-b p_{d}^{*}\right) .
$$




\section{Decentralized channel decisions under VMCl contract}

\subsection{Retailer's pricing decision}

In step 2 for a given consignment price and a given service level, chosen by the supplier in step 1 , the retailer determines the retail price, which maximizes his own expected profit. Then the retailer attains the expected profit equal to

$$
\Pi_{d, R}(p \mid w, z)=(p-w)(\mu(z)+a-b p)-c \alpha(z+a-b p) .
$$

In VMCI program we get the equivalent solution for the optimal price $p_{d}^{*}$ as in RMCI. Then for any given service level $z \in[A, B]$ and a consignment price $w>0$, the retailer's unique optimal retail price $p_{d}^{*}(w, z)$ is given by

$$
p_{d}^{*}(w, z)=\frac{\mu(z)+a+b c \alpha+b w}{2 b} .
$$

\subsection{Supplier's consignment price and service level decisions}

In step 1, knowing that the retailer's optimal price $p_{d}^{*}$ is given by (11), the supplier's aim is to set the optimal consignment price $w^{*}$ and the optimal service level $z_{d}^{*}$, which maximize his own expected profit. The supplier's profit function is equal to

$$
\Pi_{d, S}(w, z \mid p)=w(\mu(z)+a-b p)-c(1-\alpha)(z+a-b p) .
$$

The following theorem is a modification of the theorem on this subject given in Bieniek [2], but here we add the condition on $f(x)$ to have IFR property, which simplifies the assumptions of the theorem a lot. It was proved in Bieniek [2], that for any given service level $z$, the supplier's unique optimal consignment price $w^{*}(z)$ maximizing $\Pi_{d, S}(w, z \mid p)$ is given by

$$
w^{*}(z)=\frac{\mu(z)+a+b c(1-2 \alpha)}{2 b} .
$$

For further considerations we need the following lemma.

Lemma 4 For any given service level $z$ if $f($.$) satisfies the property of increasing$ failure rate (IFR) then LSR elasticity $\kappa\left(w^{*}(z)+c(1-\alpha), z\right)$ is increasing function of $z$ for all $z \in[A, B]$.

Using the above result the next theorem gives the formula for the service level and the conditions for existence of the closed-form solution.

Theorem 4 If $f($.$) satisfied the property of increasing failure rate (IFR) and$

$$
(A+a+b c(3-4 \alpha)) f(A) \geq 1
$$


then the service level $z_{d}^{*}$ is uniquely determined by

$$
\frac{\mu\left(z_{d}^{*}\right)+a-4 b c \alpha+3 b c}{2 b}=\frac{2 c(1-\alpha)}{1-F\left(z_{d}^{*}\right)} .
$$

Putting the formula for $w^{*}$ into (11) we get

$$
p_{d}^{*}\left(z_{d}^{*}\right)=\frac{3 \mu\left(z_{d}^{*}\right)+3 a+b c}{4 b} .
$$

Note the the optimal selling price in VMCI program given by (13) is independent on the share of channel cost $\alpha$. Furthermore, the consignment price $w^{*}$ is increasing function in $z$ and it is concave, which is the same as $p_{d}^{*}$. Moreover $p_{d}^{*}$ should be such that $p_{d}^{*}<p_{\max }=\frac{A+a}{b}$, which gives

$$
4 A+a-b c>1
$$

Finally, we state that in VMCI the total channel decentralized profit is in general equal to (10), which in this case gives

$$
\Pi_{d}^{*}=\frac{3 \mu\left(z_{d}^{*}\right)+3 a+b c}{16 b}\left(\mu\left(z_{d}^{*}\right)+a+3 b c\right)-c\left(z_{d}^{*}+a\right) .
$$

\section{Numerical example}

In order to illustrate the results previously obtained we proceed a numerical example. In particular we use uniformly distributed demand on the interval $[A, B]$ with $A=-3$ and $B=3$. Moreover, we set the model parameters equal to $a=35, b=1$ and $c=20$. In such a case the general assumptions and (3) are satisfied, so we can obtain the results for the centralized channel. Namely, we get $z_{c}^{*}=-1.502, p_{c}^{*}=26.656$ and $\Pi_{c}^{*}=40.556$.

In case of decentralized channel under RMCI contract Table 1 contains the optimal service level $z_{d}^{*}$, the optimal retail price $p_{d}^{*}$ and the optimal consignment price $w^{*}$ with respect to the share of channel $\cos t \alpha$, because this is a strategic parameter. Additionally, we give the values of the optimal profit for the channel and also the optimal profit for the retailer and for the supplier, separately. Here we can give the precise solutions if (4), (5) and (8) hold. For that reason $\alpha$ should be greater than approximately 0.15 by (8). Also after we assign $p_{d}^{*}$ and $w^{*}$ we have to check if $p_{d}^{*}<p_{\max }$, (4) and (5) are satisfied. For RMCI arrangement, the increasing share of the channel cost leads to the decreasing service level and for the decreasing retail price, too. The consignment price also decreases, if the share of channel cost increases.

In case of decentralized channel under VMCI contract Table 2 contains the results on the optimal quantities and the values of the maximal expected profits with respect to the share of the channel cost $\alpha$. Here we can give the precise solutions if (12) and (14) are satisfied. From (12) the share of channel cost $\alpha$ in our specific model can 
Table 1 Optimum values as a function of $\alpha$ for $\epsilon \sim U[-3,3]$ and parameters $a=35, b=1, c=20$ under RMCI contract

\begin{tabular}{lcrrrccccc}
\hline$\alpha$ & \multicolumn{1}{c}{0.2} & \multicolumn{1}{c}{0.3} & \multicolumn{1}{c}{0.4} & \multicolumn{1}{c}{0.5} & \multicolumn{1}{c}{0.6} & \multicolumn{1}{c}{0.7} & \multicolumn{1}{c}{0.8} & \multicolumn{1}{c}{0.9} & \multicolumn{1}{c}{1.0} \\
\hline$z_{d}^{*}$ & -0.5 & -0.943 & -1.262 & -1.502 & -1.688 & -1.836 & -1.956 & -2.055 & -2.137 \\
$p_{d}^{*}$ & 31.122 & 30.574 & 30.224 & 29.983 & 29.81 & 29.68 & 29.582 & 29.504 & 29.442 \\
$w^{*}$ & 24.266 & 21.444 & 18.962 & 16.656 & 14.451 & 12.31 & 10.21 & 8.137 & 6.084 \\
$\Pi_{d}^{*}$ & 21.358 & 26.046 & 28.317 & 29.482 & 30.081 & 30.372 & 30.491 & 30.509 & 30.47 \\
$\Pi_{d, R}^{*}$ & 6.078 & 7.681 & 8.627 & 9.204 & 9.561 & 9.781 & 9.916 & 9.995 & 10.038 \\
$\Pi_{d, S}^{*}$ & 15.281 & 18.365 & 19.69 & 20.278 & 20.52 & 20.591 & 20.575 & 20.514 & 20.432 \\
\hline
\end{tabular}

obtain any values from the interval $[0,1]$. In case of VMCI channel increasing share of the channel cost leads to the increasing service level and also to the increasing retail price. But the consignment price decreases when the share of channel cost increases.

We can state that for both RMCI and VMCI the retailer and the supplier do not share the net channel profit equally. In the end of the numerical analysis we present the figures with the decentralized channel profits and the loss of channel profit under both regimes. On the Figs. 1 and 2 we can observe the variability of decentralized channel profits for the retailer and the supplier. We see that under RMCI the retailer's profit increases with $\alpha$, but the supplier's profit is an increasing-decreasing function of $\alpha$. Under VMCI the retailer's profit is decreasing and the supplier's profit increasing function of $\alpha$.

Additionally, the loss of channel profit is interpreted as a percentage loss of the centralized channel profit and it is equal to $\frac{\Pi_{c}-\Pi_{d}}{\Pi_{c}}$. The variability of the profit loss with $\alpha$ in RMCI and VMCI are shown on the Fig. 3, respectively. We can state that under RMCI regime the profit loss in the beginning decreases and then increases. We see that the minimal profit loss is approximately equal to $25 \%$. It is appeared that under VMCI the profit loss is also decreasing-increasing function of $\alpha$. From the figure we see that it attains the minimum value approximately equal to $26.8 \%$.

It should be emphasized that in this numerical example we can study VMCI for any $\alpha$ but RMCI for $\alpha>0.15$. Then both regimes can be considered simultaneously only for approximately $0.15<\alpha \leq 1$. Up to $\alpha=0.5$ when the profit channel is controlled by the supplier he gives the smaller profit loss than the channel controlled by the retailer. For bigger values of $\alpha$ the conclusion is opposite. But generally joint analysis of the profit loss for both arrangements for all $\alpha \in[0,1]$ is impossible in our example. It should be remembered that we obtain closed-form solutions only for specific values of $\alpha$. It is based on the restrictive condition assuring the concavity of the expected profit functions, which is a sufficient but not necessary condition. 


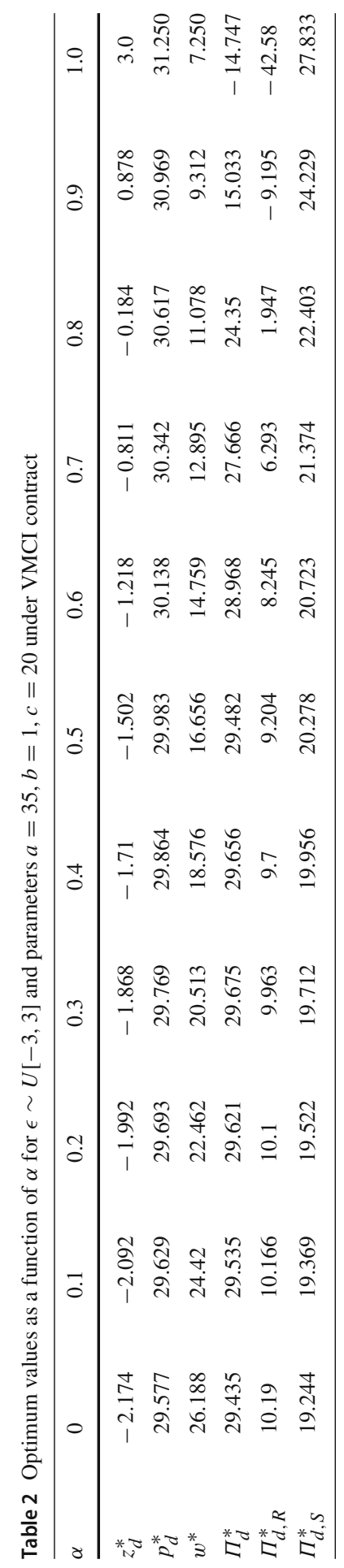



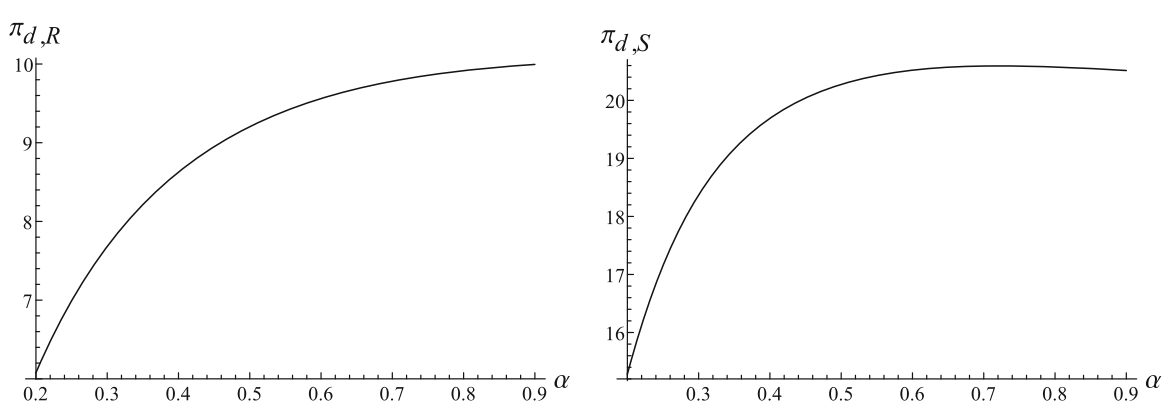

Fig. 1 Expected profit functions for the retailer (left) and the supplier (right) for RMCI with for $\epsilon \sim$ $U[-3,3], a=35, b=1, c=20$
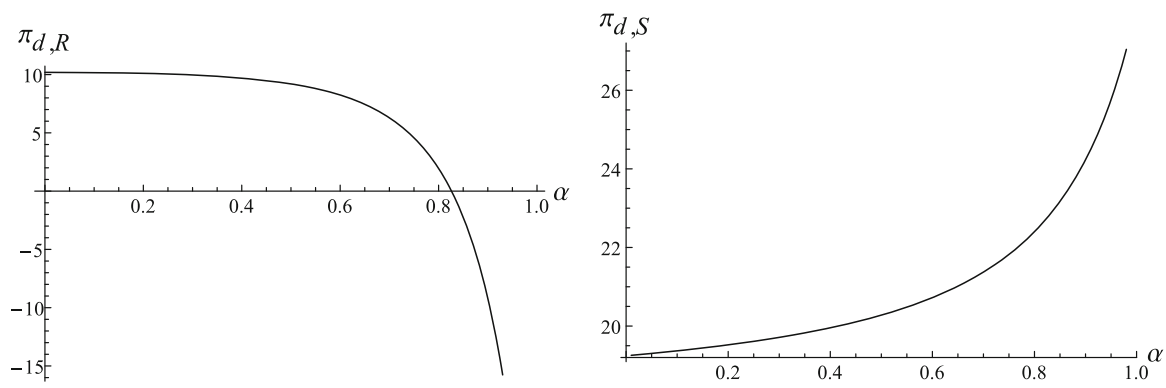

Fig. 2 Expected profit functions for the retailer (left) and the supplier (right) for VMCI with $\epsilon \sim U[-3,3]$, $a=35, b=1, c=20$

Fig. 3 Profit losses for VMCI (dashed) and RMCI (solid) for $\epsilon \sim U[-3,3], a=35, b=1$, $c=20$

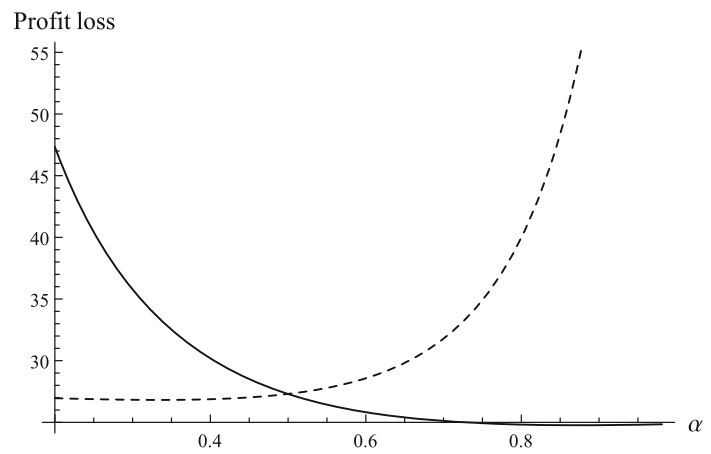

\section{Conclusions}

In this paper we analyse the problem studied in Ru and Wang [10] and Bieniek [2]. In the first mentioned paper a game-theoretic newsvendor-type model is built. In the model, the authors analyze the channel decisions and performance under two different regimes. First one is called retailer managed consignment inventory (RMCI), and the second one, vendor managed consignment inventory (VMCI). In both arrangements, the upstream supplier owns the product until it gets sold by the downstream retailer. The only difference between these programs is, which channel member makes the 
decision on the inventory level or the service level. In RMCI arrangement it is done by the retailer, and in VMCI by the supplier. The authors use special kind of stochastic demand, which is multiplicative and exponentially dependent on the price. Considering the alternative demand forms like a linear function can cause difficulties of obtaining precise solutions to the model. We overcome computational difficulties and solve the problem. Although, due to the fact that the model is mathematically complex, we have to add some restrictive assumptions under which the solutions exist.

The assumptions in the main theorems presented in Bieniek [2], in which the optimal service level is found, are extremely demanding and hard to check. We simplify them in all theorems assuming the increasing failure rate property of the distribution function. But still these assumptions assure the concavity of the expected profit function, which is obviously not a necessary condition for the existence of the unique optimal solution. However, owing to the fact of concavity, we can give the closed-form solutions, which are not evident, especially for RMCI arrangement. Perhaps one can check if the optimal solution can be derived for milder condition than concavity.

Finally, in our paper we give the numerical example, which has not been presented either in Ru and Wang [10] nor in Bieniek [2]. In the computational part of our study we use uniformly distributed demand with mean 0 . The model parameters satisfy all of the assumptions of obtained theorems. Since it is hard to approach the results analytically, we discuss the variability of the solutions based on this numerical example. The optimal values with the variable channel cost share are given in tables and the optimal channel profits are presented on figures. It appears that the demanding assumptions cause some difficulties for formulating final conclusions of the examined models. Namely, in some cases RMCI contract can be used for different values of channel cost share than VMCI contract. Due to this fact, sometimes we can only analyze the arrangements separately. Therefore in such cases we cannot compare RMCI and VMCI contracts. That is why we are not allowed to state in general who should control the supply chain: the retailer or the supplier. Finally, our numerical example shows that the properties of the additive model can be different than the properties of the multiplicative model. In a future research one can prove the properties of the additive model studied here analytically.

Open Access This article is distributed under the terms of the Creative Commons Attribution 4.0 International License (http://creativecommons.org/licenses/by/4.0/), which permits unrestricted use, distribution, and reproduction in any medium, provided you give appropriate credit to the original author(s) and the source, provide a link to the Creative Commons license, and indicate if changes were made.

\section{Appendix}

Proof of Theorem 1 As a standard procedure the first order condition requires that $z_{c}^{*}$ satisfies $\frac{\partial \Pi_{c}\left(p^{*}(z), z\right)}{\partial z}=0$. The optimal value $z_{c}^{*}$ always exists since $\left.\frac{\partial \Pi_{c}\left(p^{*}(z), z\right)}{\partial z}\right|_{z=A}<$ 0 and $\left.\frac{\partial \Pi_{c}\left(p^{*}(z), z\right)}{\partial z}\right|_{z=B}>0$. Moreover, the uniqueness of $z_{c}^{*}$ implies from $\frac{\partial^{2} \Pi_{c}\left(p^{*}(z), z\right)}{\partial z^{2}} \leq 0$, which means that the expected profit function is a concave function of $z$. The last inequality is equivalent to $\kappa\left(p_{c}^{*}(z), z\right) \geq \frac{1}{2}$. On the other hand $f(x)$ has 
IFR property, which by Lemma 1 implies that LSR elasticity $\kappa\left(p_{c}^{*}(z), z\right)$ attains its minimum value at $z=A$, which gives $\kappa\left(p_{c}^{*}(A), A\right) \geq \frac{1}{2}$. This is equivalent to the assumption (3). The proof is complete.

Proof of Lemma 2 Note that

$$
\frac{d \kappa\left(p_{d}^{*}(z)-w, z\right)}{d z}=\frac{f(z)}{2}+b\left(p_{d}^{*}(z)-w\right) \frac{d}{d z}\left(\frac{f(z)}{1-F(z)}\right),
$$

which is positive by IFR property and by assuming that $p_{d}^{*}(z)-w>0$.

Proof of Theorem 2 The quantity $z_{d}^{*}$, which maximizes $\Pi_{d, R}\left(p_{d}(z), z\right)$ can be calculated from $\frac{\partial \Pi_{d, R}\left(p_{d}(z), z\right)}{\partial z}=0$. The optimal $z_{d}^{*}$ always exists since $\left.\frac{\partial \Pi_{d, R}\left(p_{d}^{*}(z), z\right)}{\partial z}\right|_{A}>$ 0 if (4) holds. Moreover $\left.\frac{\partial \Pi_{d, R}\left(p_{d}^{*}(z), z\right)}{\partial z}\right|_{B}<0$. We claim that such point $z_{d}^{*}$ is unique by proving that the profit function $\Pi_{d, R}\left(p_{d}^{*}(z), z\right)$ is concave. The statement $\frac{\partial^{2} \Pi_{d, R}\left(p_{d}^{*}(z), z\right)}{\partial z^{2}} \leq 0$ is equivalent to $\kappa\left(p_{d}^{*}(z)-w, z\right) \geq \frac{1}{2}, z \in[A, B]$. On the other hand, by Lemma 2 LSR elasticity $\kappa\left(p_{d}^{*}(z)-w, z\right)$ is increasing in $z$ and it is enough to satisfy the previous inequality by $z=A$, which gives (5). The proof is complete.

Proof of Lemma 3 From (6) we have $w=\frac{\mu\left(z_{d}^{*}\right)+a+b c \alpha}{b}-\frac{2 c \alpha}{1-F\left(z_{d}^{*}\right)}$. We need to find the conditions under which the function $w(z)$ could be inverted. Hence, it should be strictly increasing or strictly decreasing function of $z$. We obtain

$$
\frac{d w}{d z_{d}^{*}}=\frac{1-F\left(z_{d}^{*}\right)}{b}-\frac{2 c \alpha}{1-F\left(z_{d}^{*}\right)} \frac{f\left(z_{d}^{*}\right)}{1-F\left(z_{d}^{*}\right)}
$$

Moreover by IFR property of $f($.$) we get$

$$
\frac{d^{2} w}{d\left(z_{d}^{*}\right)^{2}}=-\frac{f\left(z_{d}^{*}\right)}{b}-\frac{2 c \alpha f^{2}\left(z_{d}^{*}\right)}{\left(1-F\left(z_{d}^{*}\right)\right)^{3}}-\frac{2 c \alpha}{1-F\left(z_{d}^{*}\right)} \frac{d}{d z_{d}^{*}}\left(\frac{f\left(z_{d}^{*}\right)}{1-F\left(z_{d}^{*}\right)}\right)<0,
$$

which implies that $\frac{d w}{d z_{d}^{*}}$ is decreasing. In the first case if the condition $\left.\frac{d w}{d z_{d}^{*}}\right|_{z=A}=$ $(1-2 b c \alpha f(A)) / b<0$ is satisfied, then $\frac{d w}{d z_{d}^{*}}<0$ for all $z_{d}^{*} \in[A, B]$ and $w$ is decreasing.

In the second case, $w$ is strictly increasing if $\left.\frac{d w}{d z_{d}^{*}}\right|_{z=B}>0$. But it is impossible since $\lim _{z \rightarrow B} \frac{d w}{d z_{d}^{*}}=-\infty$. The quantity $\frac{d z_{d}^{*}}{d w}$ can be obtained from (15). The proof is complete.

Proof of Theorem 3 Since $z_{d}^{*}$ is a function of $w$, putting the formula (9) into (7) and forcing $\frac{\partial \Pi_{d, S}\left(w \mid p_{d}, z_{d}\right)}{\partial w}$ equal to 0 , we get the formula for $w^{*}$. To prove the uniqueness it is enough to note that the derivative $\frac{\partial \Pi_{d, S}\left(w \mid p_{d}, z_{d}\right)}{\partial w}$ is positive for $w<w^{*}$ and negative for $w>w^{*}$, which ends the proof. 
Proof of Lemma 4 We see that

$$
\frac{d \kappa\left(w^{*}(z)+c(1-\alpha), z\right)}{d z}=\frac{f(z)}{2}+b\left(w^{*}(z)+c(1-\alpha)\right) \frac{d}{d z}\left(\frac{f(z)}{1-F(z)}\right),
$$

which is nonnegative by IFR property. The proof is complete.

Proof of Theorem 4 The optimal quantity $z_{d}^{*}$ maximizing the profit function $\Pi_{d, S}$ satisfies $\frac{\partial \Pi_{d, S}}{\partial z}(w, z \mid p)=0$. Such $z_{d}^{*}$ always exists because $\left.\frac{\partial \Pi_{d, S}}{\partial z}(w, z \mid p)\right|_{A}=\frac{A+a-b c}{4 b}>$ 0 by the general assumptions. Moreover, $\left.\frac{\partial \Pi_{d, S}}{\partial z}(w, z \mid p)\right|_{B}=-c(1-\alpha)<0$. We claim that such a point is unique by showing the concavity of $\Pi_{d, S}(w, z \mid p)$. It is known that in this case $\frac{\partial^{2} \Pi_{d, S}(w, z \mid p)}{\partial z^{2}}$ is equivalent to

$$
\kappa\left(w^{*}(z)+c(1-\alpha)\right) \geq \frac{1}{2}
$$

for all $z \in[A, B]$. On the other hand by Lemma $2 \kappa\left(w^{*}(z)+c(1-\alpha)\right)$ is increasing in $z$, which implies that it is enough to satisfy (16) by $z=A$. The proof is complete.

\section{References}

1. Barlow, R.E., Proschan, F.: Mathematical Theory of Reliability. Society for Industrial and Applied Mathematics, Philadelphia (1996)

2. Bieniek, M.: Consignment contracting with inventory control with additive price-dependent demand. In: Evtushenko, Yu G., Yu Khachay, M., Khamisov, O. V., Kochetov, Yu A., Malkova, V.U., Posypkin, M.A. (eds): Proceedings of the OPTIMA-2017 Conference, Petrovac, Montenegro, 02-Oct-2017, published at http://ceur-wsorg, 1987:88-94 (2017)

3. Cai, J., Hu, X., Pandu, R.T., Shang, J.: Flexible contract design for VMI supply chain with servicesensitive demand: revenue-sharing and supplier subsidy. Eur. J. Oper. Res. 261, 143-153 (2017)

4. Feng, X., Moon, I., Ryuc, K.: Revenue-sharing contracts in an $N$-stage supply chain with reliability considerations. Int. J. Product. Econ. 147, 20-29 (2014)

5. Hu, W., Li, Y., Govindan, K.: The impact of consumer returns policies on consignment contracts with inventory control. Eur. J. Oper. Res. 233, 398-407 (2014)

6. Hu, W., Chen, D., Yu, H.: Benefit and risk analysis of consignment contracts. Ann. Oper. Res. 257, 641-659 (2017). https://doi.org/10.1007/s10479-015-1919-0

7. Kocabiyikoglu, A., Popescu, I.: An elasticity approach to the newsvendor with price-sensitivity demand. Oper. Res. 59, 301-312 (2011)

8. Lee, C., Chu, W.: Who should control the inventory in a supply chain? Eur. J. Oper. Res. 164, 158-172 (2005)

9. Petruzzi, N.C., Dada, M.: Pricing and newsvendor problem: a review with extensions. Oper. Res. 47, 183-194 (1999)

10. Ru, J., Wang, Y.: Consignment contracting: Who should control inventory in the supply chain? Eur. J. Oper. Res. 201, 760-769 (2010)

11. Wang, Y., Jiang, L., Shen, Z.J.: Channel performance under consignment contract with revenue sharing. Manag. Sci. 50, 34-47 (2004) 